\title{
Clinical and ultrasonographic screening for carpal tunnel syndrome in hemodialysis patients
}

\author{
Samar Tharwat ${ }^{1 \text { (i) }}$, Mohammed Kamal Nassar ${ }^{2}$ (i) \\ ${ }^{1}$ Rheumatology \& Immunology Unit, Department of Internal Medicine, Faculty of Medicine, Mansoura University, Mansoura, Egypt \\ ${ }^{2}$ Mansoura Nephrology \& Dialysis Unit (MNDU), Department of Internal Medicine, Faculty of Medicine, Mansoura University, \\ Mansoura, Egypt
}

Background: Carpal tunnel syndrome (CTS) is one of the most common complications among hemodialysis (HD) patients. This study aimed to assess the prevalence of CTS in HD patients using clinical and noninvasive ultrasound (US) criteria.

Methods: A cross-sectional, observational study was conducted on 94 HD patients to evaluate CTS manifestations and demographic, clinical, and laboratory data. The median nerve (MN) cross sectional area (CSA) (MN-CSA) was estimated by US examination at the pisiform and hamate levels. Both hands were evaluated, and the higher MN-CSA was considered.

Results: Neuropathic pain and nocturnal numbness at MN distribution were present in 40.4\% and $18.1 \%$, respectively, while Tinel's test was positive in $25.5 \%$ of patients. A MN-CSA $\geq 11.5 \mathrm{~mm}^{2}$ identified the probability of CTS with $63 \%$ sensitivity and $80 \%$ specificity. Patients with CTS had higher serum calcium $(P=0.02)$ and lower parathyroid hormone (PTH) $(P=0.02)$. CTS was frequently developed on the same side of an arteriovenous fistula. The MN-CSA had positive correlations with age, serum phosphorus, and visual analogue scale (VAS) score $(P=0.01$, 0.01 , and 0.03 respectively) and a negative correlation with PTH level $(P=0.007)$. Serum phosphorus level $(P=0.015)$ and VAS $(P=0.04)$ were the significant predictors of MN-CSA.

Conclusion: CTS appears to frequently occur in HD patients. US examination may be helpful in detection of CTS and can be an alternative to electrodiagnostic studies in HD patients.

Keywords: Carpal tunnel syndrome, Median nerve, Neuropathy, Renal dialysis, Ultrasound imaging

Received January 20, 2020; Revised April 17, 2020;

Accepted April 24, 2020

Edited by Young-Ki Lee, Hallym University, Seoul, Republic of Korea Correspondence: Samar Tharwat

Rheumatology \& Immunology Unit, Department of Internal Medicine, Faculty of Medicine, Mansoura University, Mansoura University Hospital, El Gomhouria St, Mansoura 35511, Dakahlia Governorate, Egypt. E-mail: samartharwat20oo@yahoo.com

Copyright (c 2020 by The Korean Society of Nephrology

(a) This is an open-access article distributed under the terms of the Creative Commons Attribution Non-Commercial License (http://creativecommons. org/licenses/by-nc-nd/4.0/), which permits unrestricted non-commercial use, distribution, and reproduction in any medium, provided the original work is properly cited.

\section{Introduction}

The prevalence of end-stage renal disease (ESRD) is increasing worldwide [1]. Maintenance hemodialysis (HD) therapy is the most common mode of renal replacement therapy [2]. HD patients are susceptible to many comorbid conditions, such as cardiovascular disease, anemia, and peripheral neuropathy [3].

Peripheral neuropathy is a common complication that affects $60 \%$ to $100 \%$ of chronic kidney disease (CKD) patients [4]. Despite the adequacy of dialysis, peripheral neuropathy remains a major disabling problem that has a negative impact on the health-related quality of life of such patients [4]. This may be caused by uremic neuropa- 
thy or dialysis-related amyloidosis [5].

Carpal tunnel syndrome (CTS), a specific form of peripheral neuropathy, is highly prevalent among HD patients, especially those with a long history of HD [6]. Its diagnosis is based mainly on clinical examination and electrodiagnostic testing, which is time-consuming and potentially painful [7].

Ultrasound (US) imaging has been used successfully for the evaluation of CTS [8]. It can be a considered as a first line tool for diagnosis due to its accuracy, availability, and noninvasiveness in comparison to electrodiagnostic studies [9]. Several diagnostic criteria have been suggested for the diagnosis of CTS using US [10]. Some reports suggested measuring the median nerve (MN) crosssectional area (CSA) (MN-CSA) at the pisiform or the inlet level of the carpal tunnel [11]. According to Borire et al [12], the MN-CSA correlated significantly with electrophysiological studies in HD patients. Moreover, Xin et al [13] found that the presence of CTS in HD patients can be predicted by a CSA cutoff value of $11 \mathrm{~mm}^{2}$ with a sensitivity and specificity of $79.2 \%$ and $84 \%$, respectively.

Most of the studies that evaluated the prevalence and risk factors of CTS among HD patients used a clinical diagnosis of CTS or electrodiagnostic studies. To the best of our knowledge, only a few studies have assessed the prevalence of this disorder in HD patients using US. Therefore, this study was carried out to assess the prevalence of CTS among HD patients using both clinical and US criteria and to investigate the factors associated with MN-CSA among those patients.

\section{Methods}

\section{Study patients}

This cross-sectional, observational study was conducted from October 2018 to March 2019 among 94 HD patients at Mansoura Nephrology and Dialysis Unit (MNDU), Mansoura University Hospital, Egypt. The sample size was selected as a convenience sample; all patients that met the inclusion criteria were invited to participate in the study, unless they were excluded by any of the exclusion criteria or refused to participate. All participants signed informed consent prior to their enrollment in the study. The study was approved by the Mansoura Faculty of Medicine Institutional Research Board (ap- proval number: R.20.04.828).

The inclusion criteria included patients with ESRD on HD who were 18 years of age or older and on regular HD for more than 6 months. The exclusion criteria were diabetes; hypothyroidism; connective tissue disease; history of significant hand trauma, wrist fracture, or surgery; or clinical evidence of cervical radiculopathy or polyneuropathy.

Demographic data including age and sex were collected. Height and dry body weight of all patients were measured, and body mass index (BMI) was calculated. Other clinical characteristics (e.g., the side of the fistula, the duration of HD, presence of hypertension, and therapeutic history) were collected from each patient by interview.

\section{Blood sampling and laboratory tests}

Blood samples were collected from the arteriovenous fistulae (AVF) just before starting the first HD session of a week. Routine laboratory tests were performed on the days of blood sampling using an automated analyzer.

\section{Clinical evaluation of CTS}

The participants were asked about the presence of neuropathic pain or nocturnal numbness at the distribution of the MN at the hand. The visual analogue scale (VAS) was used to quantify the pain reported by the patient, ranging from zero (no pain) to ten (maximum pain).

The hand was examined using the Tinel's test. The Tinel's test was performed by tapping on the MN at the wrist joint repeatedly, 4 to 6 times, and the presence of neuropathic pain at the MN distribution was considered to be a positive test [14].

\section{US evaluation of $M N$}

All sonographic examinations were performed by an expert rheumatologist in the field of musculoskeletal sonography having at least 5 years of experience. The rheumatologist was blinded to the clinical assessment of the patients at the time of evaluation. The US machine used was the EDAN U2 ultrasound device (Shenzhen, China) with a linear array transducer (8 to $13.4 \mathrm{MHz}$ ). A frequency of $13 \mathrm{MHz}$ was used, and the sonographic parameters were adjusted in order to obtain optimal images of the MN. 
All subjects were seated with the elbow flexed to $90^{\circ}$ and the wrist and fingers extended. Transverse images of the MN were obtained at the wrist joint at the pisiform and hamate levels (tunnel inlet). The transducer was kept perpendicular to the examined MN. No additional force was applied other than the weight of the transducer to avoid any artificial deformity of the MN. The MN-CSA was measured at the site of maximum enlargement of the

Table 1. Clinical and laboratory parameters according to the presence of carpal tunnel syndrome

\begin{tabular}{|c|c|c|c|c|}
\hline Variable & All patients $(n=94)$ & Without CTS $(n=65)$ & With CTS $(n=29)$ & $P$ value \\
\hline Age (yr) & $52.90 \pm 13.38$ & $51.7 \pm 14.6$ & $55.5 \pm 9.3$ & 0.23 \\
\hline Sex & & & & 0.1 \\
\hline Male & $60(63.8)$ & $45(69.2)$ & $15(51.7)$ & \\
\hline Female & $34(36.2)$ & $20(30.8)$ & $14(48.3)$ & \\
\hline Underlying causes of ESRD & & & & 0.83 \\
\hline Hypertensive nephrosclerosis & $42(44.7)$ & $31(47.7)$ & $11(37.9)$ & \\
\hline Chronic interstitial nephritis & $7(7.4)$ & $6(9.2)$ & $1(3.4)$ & \\
\hline ADPKD & $6(6.4)$ & $4(6.2)$ & $2(6.9)$ & \\
\hline Chronic glomerulonephritis & $4(4.3)$ & $2(3.1)$ & $2(6.9)$ & \\
\hline Chronic obstructive uropathy & $4(4.3)$ & $2(3.1)$ & $2(6.9)$ & \\
\hline Chronic pyelonephritis & $2(2.1)$ & $1(1.5)$ & $1(3.4)$ & \\
\hline Chronic graft failure & $2(2.1)$ & $1(1.5)$ & $1(3.4)$ & \\
\hline Unknown cause & $27(28.7)$ & $18(27.7)$ & $9(31.0)$ & \\
\hline Dialysis duration (mo) & $30(12-60)$ & $30(18-60)$ & $24(12-60)$ & 0.36 \\
\hline$<1$-year & $16(17.0)$ & $10(15.4)$ & $6(20.7)$ & 0.65 \\
\hline $1-5$ years & $57(60.6)$ & $39(60.0)$ & $18(62.1)$ & \\
\hline$>5$ years & $21(22.3)$ & $16(24.6)$ & $5(17.2)$ & \\
\hline Body mass index $\left(\mathrm{kg} / \mathrm{m}^{2}\right)$ & $28.35 \pm 5.6$ & $28.1 \pm 5.5$ & $28.8 \pm 5.7$ & 0.58 \\
\hline Hemoglobin (g/dL) & $10.33 \pm 1$ & $10.4 \pm 0.98$ & $10.1 \pm 1.15$ & 0.28 \\
\hline TSAT (\%) & $37(24.75-53)$ & $35(24.2-53)$ & $44(24.5-55)$ & 0.21 \\
\hline Albumin (g/dL) & $4.1 \pm 0.25$ & $4.06 \pm 0.25$ & $4.15 \pm 0.24$ & 0.14 \\
\hline Cholesterol (mg/dL) & $116(92.75-156.25)$ & $118(92.25-152)$ & $104(91.75-178.25)$ & 0.72 \\
\hline Triglycerides (mg/dL) & $86(46.75-184)$ & $76.5(66-191)$ & $100.5(59.5-157)$ & 0.86 \\
\hline Calcium (mg/dL) & $8.46 \pm 1.33$ & $8.2 \pm 1.23$ & $8.9 \pm 1.39$ & 0.02 \\
\hline Phosphorus (mg/dL) & $6.44 \pm 2.3$ & $6.2 \pm 2.28$ & $6.9 \pm 2.33$ & 0.18 \\
\hline PTH (pg/mL) & 450 (162.75-812) & $518(196-937)$ & $269(121-530)$ & 0.02 \\
\hline $\mathrm{KT} / \mathrm{V}$ & $1.30 \pm 0.33$ & $1.27 \pm 0.29$ & $1.35 \pm 0.37$ & 0.29 \\
\hline $\mathrm{CRP}(\mathrm{mg} / \mathrm{L})$ & $3.8(2.15-6.38)$ & $3.88(1.98-6.31)$ & $3.6(2.27-8.17)$ & 0.75 \\
\hline AVF side & & & & 0.04 \\
\hline The same side of CTS & $47(50.0)$ & $28(43.1)$ & 19 (65.5) & \\
\hline The opposite side of CTS & $47(50.0)$ & 37 (56.9) & $10(34.5)$ & \\
\hline Neuropathic pain at the median nerve distribution & $38(40.4)$ & $22(33.8)$ & $16(55.2)$ & 0.05 \\
\hline Nocturnal numbness at the median nerve distribution & $17(18.1)$ & $6(9.2)$ & $11(37.9)$ & 0.001 \\
\hline Positive Tinel's test & $24(25.5)$ & $9(13.8)$ & $15(51.7)$ & $<0.001$ \\
\hline Cross sectional area of the median nerve $\left(\mathrm{mm}^{2}\right)$ & $9(6-13)$ & $8(5.5-9)$ & $16(13.5-18.5)$ & $<0.001$ \\
\hline Visual analogue scale & $0(0-1)$ & $0(0-2.75)$ & $2(0-4.5)$ & 0.01 \\
\hline
\end{tabular}

Quantitative variables are expressed as mean \pm standard deviation for normally distributed data and median (interquartile range) for non-normally distributed data. Qualitative variables are expressed as number (\%). $P$ value refers to comparisons between the 2 groups, according to the presence of carpal tunnel syndrome (MN-CSA $\geq$ $11.5 \mathrm{~mm}^{2}$ ).

ADPKD, autosomal dominant polycystic kidney disease; AVF, arteriovenous fistula; CRP, C-reactive protein; CTS, carpal tunnel syndrome; ESRD, end-stage renal disease; KT/V, K: dialyzer clearance of urea, T: dialysis time, V: volume of distribution of urea; MN-CSA, median nerve cross-sectional area; PTH, parathyroid hormone; TSAT, transferrin saturation. 
MN. The CSA was assessed by tracing the margin of the hypoechoic nerve fascicles and the interior of the hyperechoic nerve sheath. Both hands were evaluated, and the higher value of the MN-CSA was considered for statistical purposes.

\section{Statistical analysis}

Data were collected, revised, verified, and analyzed using IBM SPSS Statistics version 21.0 for Windows (IBM Corp., Armonk, NY, USA) on a personal computer. Medians (interquartile range [IQR]) or means \pm standard deviation were used for all quantitative values, while numbers of cases and percentages (\%) were used to describe qualitative variables. Receiver operating characteristic (ROC) curve analysis was performed for the MN-CSA to identify the cutoff point above which CTS is likely. The cutoff point achieving the best sensitivity together with the best specificity was selected. After examining the distribution of continuous variables for normality using the Shapiro-Wilk test, the significance of differences between two groups was determined by the independent samples $t$ test for normally distributed variables or by the MannWhitney test for non-parametric variables, as appropriate. Chi-square or Fisher exact tests were used for comparison between qualitative variables, as appropriate. Univariate correlation analyses using the Spearman's test were performed to define significant factors that affected MN-CSA. Subsequently, 4 significant factors, comprising a mix of demographic, clinical, and laboratory factors, were subjected to a multivariate linear regression model using the enter approach to assess the predictors of $\mathrm{MN}$ CSA. The goodness of fit for the model was tested using chi-square goodness of fit tests. The statistical significance level was set at $<0.05$ if $P$ value is less than 0.05 .

\section{Results}

Ninety-four HD patients (60 males and 34 females) were successfully recruited to participate in the study. The mean age of the studied patients was $52.90 \pm 13$.38 years, and the mean BMI was $28.35 \pm 5.6 \mathrm{~kg} / \mathrm{m}^{2}$. Other clinical, demographic, and laboratory data of the participants are described in Table 1.

As demonstrated in Table 1, neuropathic pain and nocturnal numbness at the distribution of the MN were pres- ent in $40.4 \%$ and $18.1 \%$ of the studied patients, respectively, while Tinel's test was positive in $25.5 \%$ of patients. The median of the MN-CSA was $9 \mathrm{~mm}^{2}$ with IQR 6 to $13 \mathrm{~mm}^{2}$.

Using the clinical criteria of presence of neuropathic pain at the MN distribution together with positive Tinel's test as the reference diagnostic criteria for CTS, a ROC curve was developed to identify the threshold value of MN-CSA that can indicate the probability of CTS. An MNCSA value of $11.5 \mathrm{~mm}^{2}$ or more identifies the probability of CTS with $63 \%$ sensitivity and $80 \%$ specificity, as shown in Fig. 1.

Using MN-CSA $\geq 11.5 \mathrm{~mm}^{2}$ as a diagnostic criterion, about $31 \%$ of the patients had CTS. There was no significant difference in the age, sex, BMI, or duration of dialysis between patients with and without CTS. Regarding biochemical data, patients with CTS $(\mathrm{n}=29)$ had higher serum calcium levels $(P=0.02)$ and lower parathyroid hormone (PTH) levels $(P=0.02)$ than those without. For about two-thirds of these patients $(n=19,65.5 \%)$, the side of the AVF was the same side of MN thickening, as shown in Table 1.

As expected, patients with CTS had a higher percentage of neuropathic pain, nocturnal numbness, and positive Tinel's test at the MN distribution, a higher VAS score for pain, and a higher MN-CSA $(P=0.05,0.001,<0.001,0.01$, and $<0.001$, respectively), as shown in Table 1 .

The MN-CSA had a positive correlation with age, serum phosphorus, and VAS $(P=0.01,0.01$, and 0.03 , respectively $)$

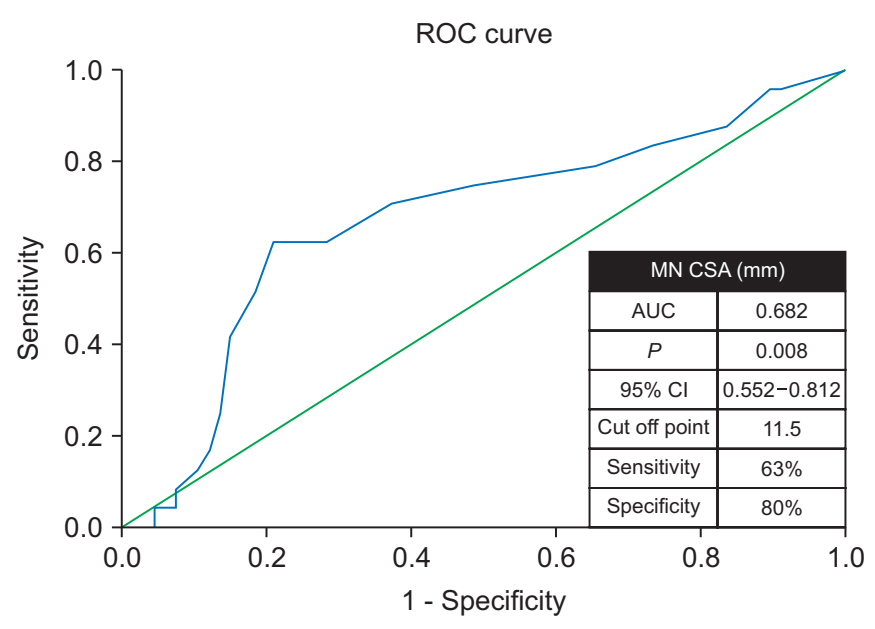

Figure 1. Receiver operating characteristic (ROC) curve analysis for ultrasonographic diagnosis of carpal tunnel syndrome using the cross-sectional area (CSA) of the median nerve (MN). AUC, area under the curve; $\mathrm{Cl}$, confidence interval. 
Table 2. Univariate correlation analyses for factors associated with the cross-sectional area of the median nerve in the study patients $(n=94)$

\begin{tabular}{lcc}
\hline \multicolumn{1}{c}{ Variable } & $\mathrm{r}^{\mathrm{a}}$ & $P$ \\
\hline Age $(\mathrm{yr})$ & 0.26 & 0.01 \\
Dialysis duration $(\mathrm{mo})$ & -0.12 & 0.30 \\
Body mass index $\left(\mathrm{kg} / \mathrm{m}^{2}\right)$ & 0.11 & 0.32 \\
Hemoglobin $(\mathrm{g} / \mathrm{dL})$ & -0.045 & 0.69 \\
TSAT $(\%)$ & 0.17 & 0.14 \\
Albumin $(\mathrm{g} / \mathrm{dL})$ & 0.08 & 0.46 \\
Cholesterol $(\mathrm{mg} / \mathrm{dL})$ & 0.068 & 0.55 \\
Triglycerides $(\mathrm{mg} / \mathrm{dL})$ & 0.05 & 0.68 \\
Calcium $(\mathrm{mg} / \mathrm{dL})$ & 0.16 & 0.14 \\
Phosphorus $(\mathrm{mg} / \mathrm{dL})$ & 0.263 & 0.01 \\
PTH (pg/mL) & -0.3 & 0.007 \\
KT/V & 0.03 & 0.79 \\
CRP (mg/L) & 0.033 & 0.77 \\
Visual analogue scale & 0.21 & 0.03 \\
\hline
\end{tabular}

CRP, C-reactive protein KT/V, K: dialyzer clearance of urea, T: dialysis time, V: volume of distribution of urea; PTH, parathyroid hormone; TSAT, transferrin saturation.

${ }^{a}$ Correlation coefficient by Spearman correlation test.

and a negative correlation with the PTH level $(P=0.007)$, as shown in Table 2. Multiple linear regression analysis was performed using the enter approach. Four potential confounders that can affect MN-CSA were included in the model. Among the studied variables, serum phosphorus level and VAS were the significant predictors of MN-CSA, as shown in Table 3.

\section{Discussion}

CTS is the most common mononeuropathy in HD patients, having considerable impact on their quality of life [15]. It is caused mainly by amyloid deposition inside the carpal tunnel [16]. Patients usually complain of an intermittent "pins-and-needles" paresthesia in the MN distribution at the hand [17]. Diagnosis is based on clinical findings, along with corroborating electrodiagnostic or ultrasonographic studies [18]. US has recently received increased attention for the evaluation of CTS as it is more widely available, noninvasive, and has lower costs [19]. US of the carpal tunnel can be useful for the assessment of CTS [20] and evaluation of dialysis-related amyloidosis [21]. To the best of our knowledge, only a few studies in the literature have evaluated US as a diagnostic tool for CTS among HD patients [21].
Table 3. Multiple linear regression analyses to identify the predictors for the cross-sectional area of the median nerve among the study patients $(n=94)$

\begin{tabular}{lcl}
\hline \multicolumn{1}{c}{ Independent variable } & Beta & $P$ value \\
\hline Constant & & 0.55 \\
Age $(\mathrm{yr})$ & 0.205 & 0.056 \\
Serum phosphorus $(\mathrm{mg} / \mathrm{dL})$ & 0.259 & 0.015 \\
PTH $(\mathrm{pg} / \mathrm{mL})$ & -0.176 & 0.1 \\
Visual analogue scale & 0.217 & 0.04 \\
\hline
\end{tabular}

PTH, parathyroid hormone.

This cross-sectional study was carried out to evaluate the prevalence of CTS among 94 HD patients through both clinical evaluation and measurement of the MNCSA using US. The associations between MN-CSA and several epidemiological, clinical, and laboratory factors were assessed. One rheumatologist with more than 5 years of experience in musculoskeletal US performed the sonographic assessment. The technique of measurement of the MN-CSA was performed as described by Mhoon et al [22] .

The frequency of clinical manifestations of CTS among HD patients was reported to range between $8 \%$ and $31 \%$ $[5,23]$. According to our results, neuropathic pain at the MN was present in $40.4 \%$, which is much higher than the percentage with positive Tinel's test (25.5\%). Using clinical manifestations plus positive Tinel's test as the reference standard, MN-CSA with a threshold of $11.5 \mathrm{~mm}^{2}$ can indicate the probability of CTS with $63 \%$ sensitivity and $80 \%$ specificity. This is consistent with a previous study carried out on 71 HD patients (22 with and 49 without CTS), which identified the cutoff value of the MN-CSA for detection of CTS as $11 \mathrm{~mm}^{2}$ with $79.2 \%$ sensitivity and $84 \%$ specificity [13].

About one-third of this cohort had MN-CSA $\geq 11.5 \mathrm{~mm}^{2}$. The high prevalence of $\mathrm{MN}$ thickening in the current study is consistent with the results of a previous study, in which 98 hands of dialysis patients were evaluated sonographically before and after transplant, resulting in the finding that dialysis was associated with MN-CSA enlargement [24]. However, contradictory to the findings of the present study, previous research that evaluated the prevalence of CTS among 385 HD patients found a lower prevalence of typical neuropathic pain (14.5\%), pathological nerve conduction test (9.6\%), and muscular atrophy (21.3\%) [19]. Accordingly, because clinical manifestations were not apparent even in many patients showing 
positive US findings, our results indicated that measuring the MN-CSA may be useful for early detection of preclinical CTS among these patients. However, this concept requires confirmation by further clinical studies.

In the present study, there was no significant difference in the prevalence of CTS (MN-CSA $\geq 11.5 \mathrm{~mm}^{2}$ ) between males and females, a finding that matches the results of Benz and colleagues [25]. However, other studies reported that the male to female ratio was $2: 1[5,26]$. In another German study that included 385 HD patients, female sex was one of the significant predictors of CTS among those patients [6]. In non-dialyzed CKD patients, CTS is wellknown to be more frequent in females (female:male $=3: 1$ ) [27]. The reduced percentage of females with CTS in HD patients could be due to either the common abnormalities occurring in ESRD in both sex or to the reduced physical activity of the patients in this study who had diminished wrist movement.

The hands affected by CTS were mostly those on the same side as the AVF. This finding is consistent with the results of Gousheh and Iranpour [28], who found that HD patients are at risk of developing CTS on the same side as the AVF. Arteriovenous shunting may either increase venous pressure and lead to nerve compression or cause ischemia of the MN due to blood stealing from the distal limb [29].

There was no significant association between MN-CSA and BMI, a finding that is not dissimilar to the results of Hammer and colleagues, who did not find any implication of weight and height on the MN-CSA [30]. However, our results contradict the findings of Franklin and Friedman, who observed an increased MN-CSA in obese patients [31]. This difference might be explained by the variance of the studied population and the diagnostic methodology of CTS used in these studies.

In this cohort, there was a direct correlation between the MN-CSA and age, however this association disappeared in multiple regression analysis. Loh et al [32] reported that the MN-CSA was higher in older patients. Despite the attenuation of the possible association between age and MN-CSA after adjustments in the present study, MN enlargement may be related to increased amyloid deposition, a known consequence of CKD, induced by the longer duration of kidney disease in these older patients [15]. Moreover, the aging process may lead to morphological changes in the myelin sheath and nerve fibers [33]. However, the reference value of the MN-CSA at different age groups still remains debatable [34].

The MN-CSA was not correlated with the dialysis vintage, a result that is in contrast to other previously published studies, which suggested the duration of dialysis treatment to be a significant risk factor for the development of CTS $[5,13,35]$. This difference can be explained by the relatively shorter duration of dialysis treatment in our cohort.

Regarding laboratory investigations, the MN-CSA had a positive correlation with serum phosphorus level and a negative correlation with the PTH level, a finding that contradicts those of Hirata and colleagues, who did not find any correlation between the time of onset of the CTS and a history of parathyroidectomy [36].

Using multiple linear regression analysis, serum phosphorus level and the VAS score were the significant predictors of MN-CSA in our patients. However, the exact mechanism of MN compression in chronic HD patients has not yet been fully explained [37]. $\beta 2$-microglobulin amyloid deposition in the carpal tunnel is the most accepted theory [38]. According to the present results, the disordered phosphorus metabolism in HD patients may play a role. Extra-skeletal calcifications are frequently encountered in HD patients and are aggravated by the persistent elevation of the calcium-phosphorus product [39]. The most common site for extra-skeletal calcifications is the blood vessels, but they may affect other sites such as periarticular tissues, myocardium, heart valves, and possibly the peripheral nerves. However, this needs to be confirmed by biopsy from the peripheral nerves.

We admit that this study has some limitations, namely the small sample size and convenience sampling method, in addition to the cross-sectional nature resulting in only a single evaluation of the MN-CSA. Unfortunately, electrodiagnostic studies were not performed. Nevertheless, the use of US in the evaluation of CTS in such patients and measurement of the MN-CSA at the entry of the carpal tunnel, which has been reported to be sensitive, are strengths of this study.

In conclusion, this study reported that CTS occurs frequently in HD patients. The hands affected by CTS were mostly those on the same side as the AVF. Among HD patients, serum phosphorus level was a predictor of the MN-CSA. US examination can be helpful in the detection of CTS and might be an alternative diagnostic modality to 
the painful and time-consuming electrodiagnostic studies. The present observations need to be confirmed in other ethnic groups and in a larger sample, and further studies are warranted to address the pathophysiologic link between this disorder and different derangements in HD patients.

\section{Conflicts of interest}

All authors have no conflicts of interest to declare.

\section{Authors' contributions}

Samar Tharwat participated in the data collection and wrote the manuscript. Mohammed Kamal Nassar participated in the conception, statistical analysis, interpretation of data and edited the manuscript. Both authors read and approved the final manuscript.

\section{References}

[1] Vanholder R, Annemans L, Brown E, et al. Reducing the costs of chronic kidney disease while delivering quality health care: a call to action. Nat Rev Nephrol 2017;13:393409.

[2] Alashek WA, McIntyre CW, Taal MW. Epidemiology and aetiology of dialysis-treated end-stage kidney disease in Libya. BMC Nephrol 2012;13:33.

[3] Pedrini LA, Winter AC, Cerino F, et al. Clinical outcomes of hemodialysis patients in a public-private partnership care framework in Italy: a retrospective cohort study. BMC Nephrol 2019;20:35.

[4] Baumgaertel MW, Kraemer M, Berlit P. Neurologic complications of acute and chronic renal disease. Handb Clin Neurol 2014;119:383-393.

[5] Kopeć J, Gadek A, Drozdz M, et al. Carpal tunnel syndrome in hemodialysis patients as a dialysis-related amyloidosis manifestation--incidence, risk factors and results of surgical treatment. Med Sci Monit 2011;17:CR505-CR509.

[6] Busch M, Schwenzky A, Franke S, Stein G, Wolf G. A Advanced glycation end products and $\beta(2)$-microglobulin as predictors of carpal tunnel syndrome in hemodialysis patients. Blood Purif 2012;34:3-9.

[7] Fowler JR, Munsch M, Tosti R, Hagberg WC, Imbriglia JE. Comparison of ultrasound and electrodiagnostic testing for diagnosis of carpal tunnel syndrome: study using a validat- ed clinical tool as the reference standard. J Bone Joint Surg Am 2014;96:e148.

[8] Ooi CC, Wong SK, Tan AB, et al. Diagnostic criteria of carpal tunnel syndrome using high-resolution ultrasonography: correlation with nerve conduction studies. Skeletal Radiol 2014;43:1387-1394.

[9] Mondelli M, Filippou G, Gallo A, Frediani B. Diagnostic utility of ultrasonography versus nerve conduction studies in mild carpal tunnel syndrome. Arthritis Rheum 2008;59: 357-366.

[10] Klauser AS, Halpern EJ, De Zordo T, et al. Carpal tunnel syndrome assessment with US: value of additional crosssectional area measurements of the median nerve in patients versus healthy volunteers. Radiology 2009;250:171177.

[11] Zyluk A, Puchalski P, Nawrot P. [The usefulness of ultrasonography in the diagnosis of carpal tunnel syndrome--a review]. Chir Narzadow Ruchu Ortop Pol 2010;75:385-391. Polish.

[12] Borire AA, Arnold R, Pussell BA, et al. Haemodialysis alters peripheral nerve morphology in end-stage kidney disease. Clin Neurophysiol 2017;128:281-286.

[13] Xin H, Hu HY, Liu B, Liu X, Li X, Li J. Ultrasound elastographic evaluation of the median nerve in hemodialysis with carpal tunnel syndrome. J Med Ultrason (2001) 2017; 44:123-131.

[14] Aroori S, Spence RA. Carpal tunnel syndrome. Ulster Med J 2008;77:6-17.

[15] Fujita K, Kimori K, Kadonishi Y, Okawa A, Ikuta Y. MRI analysis of carpal tunnel syndrome in hemodialysis patients versus non-hemodialysis patients: a multicenter case-control study. J Orthop Surg Res 2019;14:91.

[16] Scarpioni R, Ricardi M, Albertazzi V, De Amicis S, Rastelli F, Zerbini L. Dialysis-related amyloidosis: challenges and solutions. Int J Nephrol Renovasc Dis 2016;9:319-328.

[17] Katz JN, Stirrat CR. A self-administered hand diagram for the diagnosis of carpal tunnel syndrome. J Hand Surg Am 1990;15:360-363.

[18] Fujimoto K, Kanchiku T, Kido K, Imajo Y, Funaba M, Taguchi T. Diagnosis of severe carpal tunnel syndrome using nerve conduction study and ultrasonography. Ultrasound Med Biol 2015;41:2575-2580.

[19] Bueno-Gracia E, Haddad-Garay M, Tricas-Moreno JM, et al. [Diagnostic validity of ultrasonography in carpal tunnel syndrome]. Rev Neurol 2015;61:1-6. Spanish.

[20] Yamazaki T, Kawahara N, Arai K, et al. Utility of ultrasonog- 
raphy of the median nerve with a high-frequency probe for the diagnosis of dialysis-related carpal tunnel syndrome. Ther Apher Dial 2016;20:483-491.

[21] Ikegaya N, Hishida A, Sawada K, et al. Ultrasonographic evaluation of the carpal tunnel syndrome in hemodialysis patients. Clin Nephrol 1995;44:231-237.

[22] Mhoon JT, Juel VC, Hobson-Webb LD. Median nerve ultrasound as a screening tool in carpal tunnel syndrome: correlation of cross-sectional area measures with electrodiagnostic abnormality. Muscle Nerve 2012;46:871-878.

[23] Huang WH, Hsu CW, Weng CH, Yen TH, Lin JH, Lee M. Association of a high normalized protein catabolic rate and low serum albumin level with carpal tunnel syndrome in hemodialysis patients. Medicine (Baltimore) 2016;95:e4050.

[24] Carolus AE, Schenker P, Dombert T, et al. Sonographic examination of the median nerve in dialysis patients and after renal transplantation. Brain Behav 2015;5:e00406.

[25] Benz RL, Siegfried JW, Teehan BP. Carpal tunnel syndrome in dialysis patients: comparison between continuous ambulatory peritoneal dialysis and hemodialysis populations. Am J Kidney Dis 1988;11:473-476.

[26] Spertini F, Wauters JP, Poulenas I. Carpal tunnel syndrome: a frequent, invalidating, long-term complication of chronic hemodialysis. Clin Nephrol 1984;21:98-101.

[27] Phalen GS. Reflections on 21 years' experience with the carpal-tunnel syndrome. JAMA 1970;212:1365-1367.

[28] Gousheh J, Iranpour A. Association between carpel tunnel syndrome and arteriovenous fistula in hemodialysis patients. Plast Reconstr Surg 2005;116:508-513.

[29] Kumar S, Trivedi HL, Smith EK. Carpal tunnel syndrome: a complication of arteriovenous fistula in hemodialysis patients. Can Med Assoc J 1975;113:1070-1072.

[30] Hammer HB, Hovden IA, Haavardsholm EA, Kvien TK. Ultrasonography shows increased cross-sectional area of the median nerve in patients with arthritis and carpal tunnel syndrome. Rheumatology (Oxford) 2005;45:584-588.

[31] Franklin GM, Friedman AS. Work-related carpal tunnel syndrome: diagnosis and treatment guideline. Phys Med Rehabil Clin N Am 2015;26:523-537.

[32] Loh PY, Yeoh WL, Muraki S. An overview of hand postures and aging on morphological changes of the median nerve. J Physiol Anthropol 2019;38:9.

[33] Peters A. The effects of normal aging on myelin and nerve fibers: a review. J Neurocytol 2002;31:581-593.

[34] Kerasnoudis A, Pitarokoili K, Behrendt V, Gold R, Yoon MS. Cross sectional area reference values for sonography of peripheral nerves and brachial plexus. Clin Neurophysiol 2013;124:1881-1888.

[35] Gejyo F, Narita I. Current clinical and pathogenetic understanding of beta2-m amyloidosis in long-term haemodialysis patients. Nephrology (Carlton) 2003;8 Suppl:S45-S49.

[36] Hirata H, Tsujii M, Yoshida T, et al. MMP-2 expression is associated with rapidly proliferative arteriosclerosis in the flexor tenosynovium and pain severity in carpal tunnel syndrome. J Pathol 2005;205:443-450.

[37] Roquelaure Y, Ha C, Pelier-Cady MC, et al. Work increases the incidence of carpal tunnel syndrome in the general population. Muscle Nerve 2008;37:477-482.

[38] Bicknell JM, Lim AC, Raroque HG Jr, Tzamaloukas AH. Carpal tunnel syndrome, subclinical median mononeuropathy, and peripheral polyneuropathy: common early complications of chronic peritoneal dialysis and hemodialysis. Arch Phys Med Rehabil 1991;72:378-381.

[39] Block GA, Klassen PS, Lazarus JM, Ofsthun N, Lowrie EG, Chertow GM. Mineral metabolism, mortality, and morbidity in maintenance hemodialysis. J Am Soc Nephrol 2004; 15:2208-2218. 